\title{
Quelle collaboration clinico-biologique pour la prise en charge des intoxications aiguës?
}

\section{How can clinicians and biologists collaborate when dealing with acute poisonings?}

Françoise VINCENT*, Vincent DANEL

Fédération de Toxicologie Clinique et Biologique, Centre Hospitalier Universitaire de Grenoble BP 217 - 38043 GRENOBLE Cedex 9

*Auteur à qui adresser la correspondance : Françoise VINCENT, Laboratoire de Pharmacologie, Centre
Hospitalier Universitaire de Grenoble - BP 217 - 38043 GRENOBLE Cedex 9
Tél : 0476765492 - Fax :0476768938 - e-mail : FrVincent.pharmaco@chu-grenoble.fr

(Reçu le 2 octobre 2000 ; accepté le 20 octobre 2000)

\section{$R \hat{E} S U M E ́$}

A l'heure où un certain nombre de biologistes ont déjà acquis une expérience en toxicologie analytique et où d'autres, de plus en plus nombreux, souvent issus d'hôpitaux généraux, désirent étendre leur domaine d'activité à cette discipline, il semble opportun de s'interroger sur le bien fondé des analyses toxicologiques. Le nombre d'intoxications aiguës admises dans les services d'urgence est en constante augmentation et nous sommes contraints d'admettre que la demande d'analyses toxicologiques est souvent trop systématique, onéreuse, mal exploitée, et même parfois inutile. Quelle collaboration entre cliniciens et biologistes pourrait rationaliser cette activité ? Cette collaboration peut exister à deux niveaux différents :

- Un niveau incontournable qui s'inscrit dans l'activité quotidienne, au moment de la prise en charge du patient ; seul le dialogue, basé sur un échange d'informations cliniques ou biologiques, permet de justifier la demande, de définir un bilan toxicologique adapté à la situation, et de choisir la méthode à mettre en cuvre. Les résultats d'analyses doivent être commentés par le biologiste et discutés.

- En dehors du cas clinique lui-même, cliniciens et biologistes doivent entamer un travail de fond et une réflexion commune qui doivent déboucher sur des bilans d'activité, des bilans d'efficacité, des protocoles de travail et des enquêtes épidémiologiques. La collaboration, souvent élargie à d'autres disciplines, doit se prolonger bien au-delà de l'intoxication lors de l'exploitation des résultats, de la diffusion de l'information, sur le réseau Internet par exemple, ou lors de la rédaction d'articles scientifiques.

\section{MOTS-CLÉS}

Collaboration, cliniciens, biologistes, intoxications aiguës.

\section{SUMMARY}

Today, a number of biologists are highly trained in analytical toxicology. Many others, often working in general hospitals, would like to develop analytical toxicology in their own hospitals. Therefore, it is relevant to put into question the interest of toxicological analysis. The number of acute poisonings admitted to emergency departments increases constantly; we must admit that the request for toxicological analysis is far too systematic, expensive, and even sometimes useless! Which kind of collaboration between clinicians and biologists could optimize toxicological analysis? Such collaboration might occur at least at two levels :

- The first level takes place whenever a patient is admitted to the hospital. A proper exchange of clinical and biological information is the only means to justify the analysis, to define a toxicological screen suited to the clinical situation, and to choose the best analytical method. The results of the analysis should be reported by the biologist and possibly discussed with the clinician.

- Apart from clinical cases, the clinician and the biologist should be working together, combining specific skills in a common reflection which should lead to workload and efficiency assessment, working procedures definitions and epidemiological surveys. Such collaboration, often extended to other medical and biological fields, should go far beyond the acute intoxication itself, encompassing the study of the analytical results, the diffusion of information on the Internet or by means of scientific paper submission.

\section{KEY-WORDS}

Collaboration, clinicians, biologists, acute intoxication. 


\section{Introduction}

L'intoxication aiguë la plus fréquente actuellement est une intoxication volontaire, admise dans un service d'accueil des urgences 2 à 3 heures après une ingestion aiguë de médicaments, psychotropes en particulier, de gravité modérée et dont l'évolution est favorable dans la majorité des cas. Les tendances de ces dix dernières années sont une moindre gravité des intoxications aiguës, médicamenteuses en particulier, et un net "glissement" des admissions, des services de réanimation vers les services d'urgence. Ainsi, les services d'accueil des urgences sont confrontés à une charge de travail "toxicologique" de plus en plus lourde, à l'origine d'une demande trop systématique d'analyses spécialisées de la part des médecins les moins rompus à cette discipline. Les modalités de prise en charge des intoxications aiguës ont, elles aussi, évolué. La primauté du traitement symptomatique est admise par tous : correction d'une détresse respiratoire, d'un état de choc, traitement de convulsions, gestes qui ne peuvent attendre les résultats parfois tardifs de l'analyse toxicologique. L'épuration digestive par lavage gastrique ou charbon activé n'est plus systématique, l'épuration rénale a peu d'indications, l'hémodialyse est exceptionnelle, l'hémoperfusion a disparu. Quelques antidotes ont montré leur intérêt et leur efficacité en urgence : naloxone, flumazénil, hydroxocobalamine, $\mathrm{N}$-acétylcystéine, fragments Fab antidigitaliques, fragments $\mathrm{F}(\mathrm{ab}) 2$ anti-vipérin, bleu de méthylène, etc...

Traiter de la collaboration entre le clinicien et l'analyste, c'est répondre d'abord à la question de la place de l'analyse toxicologique dans la démarche diagnostique et thérapeutique d'une intoxication aiguë, dans le contexte actuel brièvement rappelé ci-dessus. Une véritable collaboration doit être bien plus qu'une simple prestation de service : c'est la mise en commun de compétences différentes pour améliorer la prise en charge d'une intoxication aiguë. Quand un clinicien demande une "recherche globale", un "screening", sans se préoccuper réellement des résultats, quand un analyste rend un résultat sans connaître l'état de l'intoxiqué, peut-on parler de collaboration ? La collaboration estelle réellement nécessaire au quotidien ? Le clinicien a$\mathrm{t}$-il besoin de la compétence du biologiste devant une intoxication par benzodiazépines sans gravité ? Le biologiste a-t-il besoin de la compétence du clinicien pour mettre en œuvre la technique la mieux appropriée à la recherche demandée ? La collaboration clinico-biologique n'est donc pas un dogme incontournable de la prise en charge d'une intoxication aiguë. Sans paradoxe aucun, la collaboration, au sens vrai du terme, n'est pas indispensable au quotidien. Cette affirmation n'exclut pas pour autant un échange nécessaire d'informations, même devant une intoxication à priori "banale", afin d'éviter tout gaspillage d'énergie, de temps et d'argent par le biologiste qui doit décider d'un protocole analytique adapté à la situation, et, pour éliminer éventuellement tout malentendu auprès du clinicien confronté à un résultat qui peut dans certains cas paraître "surprenant"!

Par contre, certaines circonstances nécessitent une véritable collaboration. On peut distinguer ainsi trois "moments" de collaboration nécessaire et souvent indispensable, qui peuvent se situer avant, pendant et après l'intoxication.

\section{Collaboration avant l'intoxication}

C'est la collaboration "en amont". Elle doit permettre au vu des données épidémiologiques nationales, régionales et locales de définir l'intérêt de l'analyse toxicologique et le rôle du laboratoire dans la prise en charge des intoxications aiguës. Elle consiste à étudier la fréquence des intoxications, à identifier les produits le plus souvent en cause, à définir des échelles de toxicité comparée des différentes molécules dans une même famille pharmacologique ou chimique, à identifier des facteurs de risque et de gravité (terrain, associations, doses, voies d'administration....), et enfin, à mesurer l'impact réel de l'analyse sur la conduite diagnostique et thérapeutique : signalons que, à ce jour, très peu de travaux rigoureux et objectifs traitent de ce dernier aspect.

Cette collaboration peut déboucher sur des consensus définissant des "bilans toxicologiques" répondant au mieux aux besoins cliniques quotidiens. Elle pourra préciser les contraintes de l'analyse en terme de praticabilité, de délai de réalisation, de coût, et de fiabilité du résultat ; elle permettra aussi de juger des avantages et inconvénients respectifs de l'aspect qualitatif ou quantitatif d'une analyse. Ainsi par exemple, doit-on encore rechercher des benzodiazépines sans détecter des molécules comme la venlafaxine, le citalopram, la clozapine ou l'olanzapine, dont on connaît le potentiel toxique ? Ces discussions entre le clinicien et l'analyste doivent être renouvelées régulièrement de façon à tenir compte de l'évolution du profil des intoxiqués, de la mise sur le marché de nouvelles molécules ainsi que des progrès technologiques. A ce niveau, la collaboration clinico-biologique doit déboucher sur l'ouverture de pistes de travail à proposer aux différentes commissions spécialisées dans le domaine de la toxicologie d'urgence. Cette collaboration "en amont" est indispensable pour mener à bien des études épidémiologiques et pour conduire ensemble des études prospectives en toxicologie clinique et analytique. 


\section{Collaboration à l'admission d'une intoxication aiguë}

L'analyse toxicologique permet classiquement :

- de confirmer une intoxication suspectée,

- d'exclure une hypothèse toxique ou de poser un diagnostic différentiel d'intoxication,

- d'évaluer la gravité d'une intoxication,

- de surveiller une thérapeutique, d'en mesurer l'efficacité.

Au-delà des stéréotypes, comment faut-il situer la collaboration clinico-biologique à chacune de ces étapes ?

\section{Confirmer une intoxication suspectée}

L'anamnèse, l'interrogatoire du patient, des témoins, de sa famille, l'examen clinique, l'évolution des premières heures, les examens biologiques de base, sont souvent suffisants pour "accepter" le diagnostic d'intoxication sans aucun résultat d'analyse toxicologique et prendre les premières mesures thérapeutiques. Exiger par exemple une recherche de benzodiazépines n'est pas justifiée en routine, sauf dans le cadre d'une éventuelle étude épidémiologique. Certains éléments cliniques et biologiques sont très évocateurs de telle ou telle intoxication (tableau I).

Par contre, chaque fois que les signes cliniques ou biologiques ne sont pas conformes à l'anamnèse, chaque fois que l'évolution n'est pas conforme à l'intoxication supposée, une collaboration entre le clinicien et l'ana- lyste doit s'instaurer pour envisager d'autres hypothèses toxiques. Dans ce cas, le clinicien doit informer le biologiste des anomalies biologiques ou cliniques observées afin d'orienter la recherche. Un complément d'enquête concernant le patient (identité, profession, circonstances et lieu de découverte, antécédents médicaux et psychiatriques) peut apporter de nouveaux éléments. Une fois établie la liste des toxiques potentiels à rechercher, il reste à choisir la méthode analytique qui va être utilisée.

Les méthodes immunochimiques automatisées sont particulièrement bien adaptées à l'urgence du fait de leur grande praticabilité d'utilisation (formation rapide du personnel), de leur rapidité (résultat rendu en 30 minutes), de leur bonne sensibilité et de leur fiabilité (à condition d'en connaître parfaitement les limites). En ce qui concerne les molécules pour lesquelles il existe un coffret réactif pour dosage spécifique, il n'y a aucun problème quelque soit le principe de la méthode utilisée (EMIT ou FPIA). L'utilisation des réactifs à destinée purement toxicologique (identification de classes de médicaments ou de stupéfiants) est plus délicate pour l'interprétation des résultats ; en effet, celle-ci fait intervenir :

- la notion de différences d'affinité, vis à vis d'un même anticorps, entre les différentes molécules d'une même famille : certaines molécules sont moins bien reconnues que d'autres, du fait parfois d'une très légère modification de structure, d'où le risque de résultats faussement négatifs ( exemple classique de certaines benzodiazépines).

Tableau I : Exemples de toxiques à évoquer devant des anomalies biologiques.

\begin{tabular}{|c|c|}
\hline Antivitamines $\mathrm{K1}$ & taux de prothrombine $<60 \%$, INR $>1,5$ (facteur $V$ normal) \\
\hline Antirouilles & hypocalcémie \\
\hline Aspirine $3<x_{0}$ & alcalose respiratoire, acidose métabolique, déshydratation \\
\hline Chloroquine & hypokaliémie \\
\hline Colchicine & hémoconcentration, acidose, TP effondré, CIVD, hyperleucocytose \\
\hline Cyanures & acidose métabolique, hyperlactatémie \\
\hline Digitaliques & hyperkaliémie \\
\hline Ethylène glycol & $\begin{array}{l}\text { acidose métabolique non lactique, non cétonémique, hyperosmolalité, } \\
\text { trou anionique élevé, hypocalcémie }\end{array}$ \\
\hline $\begin{array}{l}\text { Hypoglycémiants } \\
\text { (éthanol chez le petit enfant), }\end{array}$ & hypoglycémie \\
\hline Méthanol & $\begin{array}{l}\text { acidose métabolique non lactique, non cétonémique, hyperosmolalité, } \\
\text { trou anionique élevé }\end{array}$ \\
\hline Paracétamol $=1+x^{2}$ & cytolyse hépatique \\
\hline Paraquat & $\begin{array}{l}\text { insuffisance rénale aiguë organique et cytolyse hépatique } \\
\text { puis hypoxie réfractaire }\end{array}$ \\
\hline Pesticides organophosphorés & chute des cholinestérases plasmatiques \\
\hline Xanthines et bêta-mimétiques & hypokaliémie, hyperleucocytose, hyperglycémie et acidose lactique \\
\hline
\end{tabular}


- la notion de réactions croisées par suite d'une parenté structurale conduisant à de faux positifs (exemple classique de la carbamazépine ou des phénothiazines dont la structure tricyclique interfère avec les réactifs du kit antidépresseurs tricycliques).

- la notion de résultat purement qualitatif (positif ou négatif par rapport à un seuil de positivité) ou de résultat semi-quantitatif, pour lequel il faut être très prudent, mais qui peut être potentiellement très intéressant : dans le cas d'une crise d'épilepsie, une recherche d'antidépresseurs supérieure à $1000 \mu \mathrm{g} / \mathrm{l}$ est suffisante pour confirmer en urgence un surdosage en antidépresseurs tricycliques.

L'immunochimie présente deux inconvénients majeurs, son coût et son domaine d'application réduit aux molécules pour lesquelles des réactifs ont été développés. Le biologiste qui ne dispose que d'un automate d'immunochimie se trouve ainsi démuni lorsqu'il est confronté par exemple à une intoxication par la chloroquine, par le méprobamate, par un neuroleptique ou par un antidépresseur non tricyclique. Il faut bien reconnaître que la facilité d'utilisation nous conduit à des situations quelque peu absurdes, où le biologiste procède à des recherches systématiques de benzodiazépines et néglige sur le plan analytique des molécules d'intérêt toxicologique. On peut regretter, en tant qu'utilisateur, que les développements d'applications se fassent plus en fonction de considérations économiques (marchés américains) qu'en fonction de critères de toxicité. Le clinicien doit être averti de toutes ces difficultés d'ordre technique : il doit savoir que les antidépresseurs de nouvelle génération ne sont pas identifiés avec le coffret des antidépresseurs, que le zolpidem et la zopiclone, souvent assimilées à tort à des benzodiazépines, ne sont pas reconnus par l'anticorps antibenzodiazépines, ou encore que la buprénorphine est une exception dans la famille des morphiniques (tableau II). Afin d'éviter tout malentendu, les résultats du laboratoire doivent être accompagnés d'une interprétation par le biologiste.

Pour combler les lacunes de l'immunochimie, ce dernier peut avoir recours à d'autres méthodes dites séparatives, les méthodes chromatographiques. Il s'agit de méthodes dites spécifiques, sensibles, se prêtant à des applications quantitatives ou qualitatives (screening avec possibilité d'utiliser des bibliothèques de spectres) et leur domaine d'application est illimité. Ces méthodes utilisent un matériel plus sophistiqué, sous la responsabilité de personnel spécialisé. Les plus classiques sont la spectrométrie de masse (SM) et la chromatographie liquide couplée à un détecteur à barrette de diodes (CLHP-DAD). Ces techniques ont une place indéniable en toxicologie d'urgence puisque leur domaine d'application est complémentaire de celui de l'immunochimie : la CLHP-DAD est performante pour les antidépresseurs y compris ceux de nouvelle génération, les benzodiazépines, les neuroleptiques et de nombreux autres médicaments; elle fonctionne en routine dans la plupart des laboratoires. La spectrométrie de masse permet une identification formelle des molécules et de leurs métabolites ; son usage est le plus souvent différé. Ces méthodes restent cependant lourdes et consommatrices de temps ; leur mise en application dépend des moyens de chaque laboratoire.

A ce niveau, se pose le problème de l'urgence de la réalisation des analyses plus complexes ; il est très difficile de répondre à cette question dans l'absolu. La réponse dépend de nombreux facteurs tels que :

- la nécessité d'instaurer un traitement spécifique antidotique à renouveler dans le temps (utilité du dosage des glycols et du méthanol en chromatographie en phase gazeuse en urgence et en garde).

- l'urgence de la prise en charge thérapeutique du patient : on ne doit pas attendre les résultats du laboratoire pour traiter une intoxication par les cyanures, l'analyse peut être différée!

- l'expérience du clinicien qui prend en charge le patient ; celle-ci influence souvent son degré d'exigence dans le sens d'une économie de santé ou au contraire d'une prescription abusive d'analyses.

- les habitudes du laboratoire, de ses moyens en personnel, de ses horaires d'ouverture, de l'existence d'un système de garde avec du personnel qualifié. En terme d'efficacité et de fiabilité des résultats, il est parfois plus prudent de reporter au lendemain une analyse complexe qui nécessite un certain "doigté" de la part de celui qui va la prendre en charge. Quelle que soit la puissance de l'outil analytique, le biologiste doit toujours rester critique face à ses résultats ; il doit faire part de ses doutes au clinicien.

- le caractère d'urgence peut relever aussi de contraintes administratives, décision de sortie du patient ou de transfert par exemple. Il peut être lié à des considérations économiques : une analyse positive confirmant un diagnostic toxicologique peut éviter d'autres investigations plus coûteuses. Tout ceci est discutable et peut alimenter le débat.

L'observation de différents laboratoires français de toxicologie montre une très grande disparité dans leur mode de fonctionnement, plus particulièrement en garde ; il serait intéressant d'en connaître le retentissement réel au niveau de la qualité de la prise en charge des patients.

La collaboration clinico-biologique est particulièrement précieuse devant une intoxication rare ou peu documentée dans la littérature scientifique. La seule 
impression clinique ou l'intime conviction ne sont pas suffisantes ; il est important alors de tout faire pour documenter avec rigueur une telle intoxication afin d'informer la communauté scientifique à bon escient. On peut même avancer l'idée que c'est un devoir pour un service spécialisé et un laboratoire bien équipé de documenter correctement ces intoxications rares ou mal connues. Dans ce cas, la collaboration peut s'établir à distance, dès lors qu'il s'agit bien de la mise en commun de compétences complémentaires.

Dans certains cas enfin, l'analyse toxicologique peut s'imposer pour des raisons médico-légales ; une suspicion de soumission chimique en est un exemple. L'importance de conserver les prélèvements pour une analyse ultérieure doit être soulignée, en particulier dans les cas douteux et/ou rares afin de ne pas perdre une chance de faire un diagnostic exact et rigoureux, même à posteriori.

\section{Exclure une hypothèse toxique ou poser un dia- gnostic différentiel d'intoxication}

De nombreux états cliniques peuvent simuler une intoxication aiguë. La notion de diagnostic différentiel doit toujours être présente à l'esprit du clinicien et du biologiste. Là encore, guidé par les symptômes cliniques et biologiques, le biologiste peut orienter sa recherche vers l'élimination de telle ou telle molécule. Dans d'autres circonstances plus atypiques et en l'absence de contexte d'intoxication, il est impossible d'être exhaustif dans une démarche analytique, la liste des produits toxiques étant illimitée. Certains cliniciens, en prescrivant des bilans que l'on peut qualifier de "tous azimuts", n'ont pas conscience de l'ampleur du travail demandé. Un tel investissement du laboratoire en temps et en argent est-il bien raisonnable ? Il apparaît donc essentiel que le clinicien soit informé du coût et de la durée de certaines analyses pour justifier sa demande (tableau II). Le laboratoire de toxicologie est parfois sollicité tardivement dans le cas d'un diagnostic différentiel ; en fonction du délai, la nature des prélèvements à analyser peut être remise en cause par le biologiste (un prélèvement de sang fait au $5^{\text {ime }}$ jour ne présente plus d'intérêt!). Sachant que le sang reste cependant le milieu biologique le plus intéressant en termes de corrélations clinico-biologiques et que la majorité des médicaments n'y sont détectés que pendant quelques heures, le biologiste tentera de retrouver quelques prélèvements faits à l'entrée et stockés dans un autre laboratoire; si sa recherche est infructueuse, il réclamera des urines ou éventuellement d'autres milieux d'excrétion (phanères) qui offrent une plus grande probabilité d'identification.
Un cas particulier est le diagnostic de l'état de mort cérébrale : le clinicien doit pouvoir éliminer avec certitude, certaines intoxications comme les barbituriques, les carbamates médicamenteux ou le chloralose, avant d'affirmer le décès.

\section{Évaluer la gravité d'une intoxication}

De nombreux "marqueurs" paracliniques simples permettent d'évaluer la gravité d'une intoxication : hyperkaliémie des digitaliques ou hypokaliémie de la chloroquine par exemple, acidose métabolique des alcools et glycols, élargissement du complexe QRS avec les médicaments à effet stabilisant de membrane, etc... Peu de taux sanguins de toxiques sont de véritables marqueurs de la gravité d'une intoxication : paracétamol, aspirine, lithium, alcools et glycols, paraquat... La corrélation entre concentrations sanguines et effets toxiques, entre concentrations sanguines et tissulaires, n'est pas une généralité mais plutôt une exception. La connaissance de la toxicocinétique du produit a alors une importance toute particulière pour donner à ces taux sanguins toute leur valeur; seule une collaboration bien comprise entre clinicien et biologiste permet de garantir la valeur informative de l'analyse toxicologique. Que faire d'une paracétamolémie à la $2^{\text {¿me }}$ heure ? d'un taux sanguin de salicylés sans tenir compte de la forme galénique du médicament ou de l'état d'hydratation du patient ? d'un taux d'éthylène-glycol à la $12^{\text {eme }}$ heure sans tenir compte de l'importance des biotransformations ? Le clinicien et le biologiste sont à l'évidence co-responsables de la pertinence du résultat.

\section{Surveiller une thérapeutique, en mesurer l'effi- cacité}

L'analyse toxicologique a rendu dans ce domaine de très grands services à la toxicologie clinique. Il eut été impossible d'évaluer l'intérêt du sirop d'ipéca, du lavage gastrique ou du charbon activé, de l'épuration rénale ou extra rénale, sans l'apport de l'analyse toxicologique. Il serait maintenant impensable de proposer un nouveau traitement épurateur sans le valider par l'analyse toxicologique. Encore faut-il que les questions soient bien posées. La seule observation d'une baisse des taux plasmatiques peut donner une fausse impression d'efficacité sans amener la preuve d'une véritable épuration, dont on souhaite qu'elle soit en plus supérieure aux capacités d'épuration spontanée de l'organisme. La confrontation des connaissances et des idées, des protocoles d'étude cinétique menés avec rigueur, sont seuls à même de répondre correctement aux questions posées. Les résultats de l'analyse toxicologique ont une influence directe sur la conduite thérapeutique 


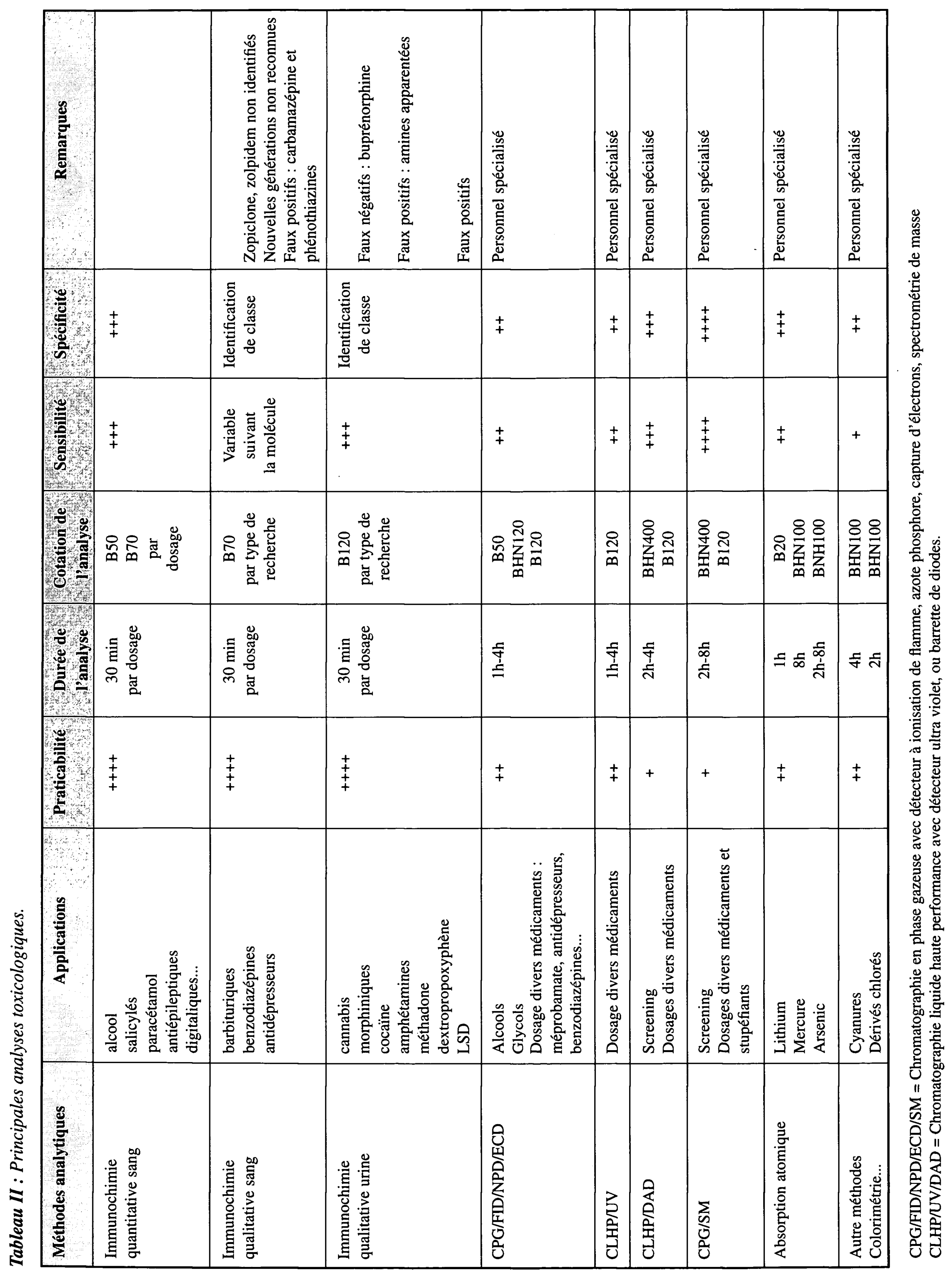


dans quelques cas : paracétamol et $\mathrm{N}$-acétylcystéine, discussion d'une épuration extrarénale dans l'intoxication par lithium, méthanol ou éthylène-glycol.

\section{Collaboration après l'intoxication}

C'est souvent le temps de l'analyse à distance de prélèvements stockés en vue d'une étude plus complète de l'intoxication. La collaboration va permettre l'étude à distance et à froid de la cinétique d'une intoxication et des relations éventuelles entre concentrations sanguines mesurées et effets observés, ainsi que la comparaison avec les données de la littérature. Ce peut être aussi le temps d'une collaboration élargie à d'autres cliniciens et d'autres biologistes, chaque fois en fonction de l'expérience et de la compétence des uns et des autres. On peut citer ici l'intérêt tout particulier des échanges d'information qui peuvent se faire facilement sur le réseau Internet par le moyen de forums ou de listes de diffusion.

\section{Conclusion}

Un dialogue entre cliniciens et biologistes doit être instauré lors de la prise en charge d'une intoxication aiguë pour éviter de tomber dans le piège inutile et onéreux du dépistage systématique ; les résultats de l'analyse toxicologique doivent être discutés et commentés pour en retirer une juste interprétation. Au-delà du quotidien, la collaboration clinico-biologique doit prendre une autre dimension, plus "académique", par la mise en commun de compétences spécifiques et par l'élargissement de la réflexion à d'autres disciplines (médecine légale, chimie analytique, épidémiologie...); elle doit prendre une dimension nationale par une plus grande collaboration des sociétés savantes, mais aussi internationale grâce à des travaux publiés ensemble dans des revues de renommées internationales.

\section{Références}

1. Bryson P. The role of the laboratory. Comprehensive review in toxicology for emergency clinicians. Taylor \& Francis, $1996:$ 47-60.

2. Gaillard Y. Méthodes analytiques. In : Kintz P., ed. Toxicologie et pharmacologie médico-légales. Elsevier, $1998: 67-110$.

3. Galliot-Guilley M., Fompeydie D. Dosage des médicaments pour le diagnostic et la surveillance des intoxications aiguës. Rev. Prat. 1997 ; 47 : 721-5.

4. Jaeger A. Epuration des toxiques au cours des intoxications aiguës : méthodes et indications. In : Jaeger J., Vale J.A. Intoxication aiguës. Elsevier, 1999 : 162-78.

5. Jaeger A., Mangin P., Sauder P., Koperschmitt J. Intérêt et limites de la recherche des toxiques en urgence. L'analyse toxicologique doit être ciblée par le clinicien. Rev. Prat. Med. Gen. 1991 ; 5 : 287-92.

6. Kintz P., Dumestre-Toulet V. L'analyse toxicologique hospitalière. In : Danel V., Barriot P., ed. Intoxications aiguës en réanimation. Arnette, 1999 : 97-110.

7. Lapostolle F., Gourlain H., Adnet F., Lapandry C. Identification des toxiques et dosage. In : Médecine d'urgence. $41^{\mathrm{emc}}$ Congrès national d'anesthésie et de réanimation. Elsevier, 1999 : 67-79.

8. Lheureux P., Askenasi R., Maes V. Du bon usage du laboratoire de toxicologie. 2e partie : utilité clinique et interprétation des résultas. Réan. Urg. 1996 ; 5 : 341-52.

9. Lheureux P., Maes V. Indications et interprétation des analyse toxicologiques. In : Jaeger J., Vale J.-A. Intoxication aiguës. Elsevier, 1999 : 61-77.

10. Lheureux P., Oleffe V. Apport du laboratoire et des données toxicologiques. In : Cours supérieurs d'urgence : Arnette, 1999 : 79-92.

11. Taboulet P. Place de l'analyse toxicologique aux urgences. La Revue des SAMU. 2000 ; 22 : 54-7.

12. Taboulet P., Azoyan P. Place des examens complémentaires dans la démarche thérapeutique de l'urgence toxicologique. Réan. Soins intens. Méd. Urg. 1994 ; 10 : 12-7.

13. Wennig R. Laboratory diagnosis of poisonings. In : Descotes J., ed. Human Toxicology. Elsevier, 1996 : 25236. 